\title{
Oral anticoagulant therapy in atrial fibrillation older patients with previous bleeding
}

\author{
Mario Bo, Francesco Giannecchini, Martina Papurello, Enrico Brunetti \\ Section of Geriatrics, Department of Medical Sciences, University of Turin, Città della Salute e della Scienza, Molinette \\ Hospital, Turin, Italy
}

\begin{abstract}
Oral anticoagulant therapy (OAT) with direct oral anticoagulants (DOACs) is the established treatment to reduce thromboembolic risk in patients with atrial fibrillation (AF). Bleeding risk scores are useful to identify and correct factors associated with bleeding risk in AF patients on OAT. However, the clinical scenario is more complex in patients with a previous bleeding event, and the decision about whether and when starting or re-starting OAT in these patients remains a contentious issue. Major bleeding is associated with a subsequent increase in both short- and long-term mortality, and even minimal bleeding may have a prognostic importance because it frequently leads to disruption of antithrombotic therapy. There is an unmet need for guidance on how to manage antithrombotic therapy after bleeding has occurred. While waiting for observational and randomized data to accrue, this paper offers a perspective on managing antithrombotic therapy after bleeding in older patients with AF.
\end{abstract}

Correspondence: Mario Bo, Section of Geriatrics, Department of Medical Sciences, University of Turin, Città della Salute e della Scienza, Molinette Hospital, Corso Bramante 88, 10126 Torino, Italy. Tel. +39.011.6335006 - Fax +39.011.6335263.

E-mail: mario.bo@unito.it

Key words: elderly; atrial fibrillation; oral anticoagulant therapy; bleeding

Contributions: all the Authors contributed equally to study conception and design, manuscript drafting and final approval and agree to be accountable for all aspects of the work.

Conflict of interest: the authors declare no conflict of interest.

Received for publication: 19 February 2020.

Accepted for publication: 21 February 2020.

${ }^{\circ}$ Copyright: the Author(s), 2020

Licensee PAGEPress, Italy

Monaldi Archives for Chest Disease 2020; 90:1552

doi: 10.4081/monaldi.2020.1252

This article is distributed under the terms of the Creative Commons Attribution Noncommercial License (by-nc 4.0) which permits any noncommercial use, distribution, and reproduction in any medium, provided the original author(s) and source are credited.

\section{Introduction}

Bleeding is a frequent complication of the management of patients with atrial fibrillation (AF). Randomized trials have shown a risk of major bleeding around 2-5\% per year in patients with AF treated with oral anticoagulant therapy (OAT) [1]. Major bleeding is associated with a subsequent increase in both short- and longterm mortality [2]. Moreover, discontinuation of antithrombotic drugs and prothrombotic responses following a bleeding may lead to an increased rate of thrombotic events due to the progressive recovery of platelet function and coagulation activity. It is well known that patients who are more likely to suffer from bleeding complications of antithrombotic therapy also tend to be at higher risk of thrombotic events [3]. Ageing is associated with a progressive increase of bleeding and thrombotic risk. In a population study on 359,166 people without cardiovascular (CV) disease not receiving antithrombotic therapies, the annual risk of major gastrointestinal bleeding was $0.6 \%$ in men and women aged $30-39$ years and $2.3 \%$ and $1.6 \%$ in men and women, respectively, aged $70-79$ years [4]. The annual risk of major intracranial bleeding was $0.03 \%$ in people aged $30-39$ years, and $0.3 \%$ and $0.2 \%$ in men and women, respectively, aged 70-79 years [4]. There are few data on bleeding risk in the oldest-old patients with AF. A matched cohort study including AF patients treated with vitamin $\mathrm{K}$ antagonists (VKAs) at a thrombosis service demonstrated that bleeding risk is only mildly increased in people aged 90 years and over compared with patients aged 70 to 79 years, whereas patients in their $80 \mathrm{~s}$ had a risk of bleeding comparable with that of patients in their 70s [5]. Interestingly, there was a steeper increase in the risk of thrombotic events in those aged 90 years and over compared with patients in their 70s and 80s [5]. Moreover, the risk of bleeding in these very old patients was not significantly affected by the quality of warfarin therapy [5]. These recent findings are in keeping with a previous study which showed that, among 4093 patients aged 80 years and over receiving VKAs for AF or pulmonary embolism, the annual rate of bleeding was $2.22 \%$ in those aged $\geq 85$ years compared with $1.71 \%$ in those aged $<85$ years [6]. Moreover, $82.2 \%$ of bleeding events occurred in patients with an international normalized ratio (INR) in the range 2.0-3.0 [6]. Altogether, these findings suggest that there is a mild increase in the bleeding risk in advanced age, and that OAT, rather than directly "causing", makes evident underlying silent bleeding. This is one of the main reasons why current scores recommended for estimating bleeding risk have demonstrated similar, albeit modest, performance in predicting OAT-associated bleeding in patients with AF [7].

In daily clinical practice older AF patients with a previous bleeding represent a common clinical scenario. Whether and when to start or re-start OAT in these patients is a complex decision, 
involving a careful evaluation of potential harm vs benefit ratio. Patients with recent bleeding have been excluded from most randomized trials of antithrombotic therapy and rigorous evidence to inform decisions is scarce. Clearly, balancing the risks of further bleeding against potentially fatal thrombotic events is critical for decisions about if and when to start or restart antithrombotic therapy after bleeding. In this paper we will review evidences to inform clinical decisions in this setting of patients.

\section{Methods}

Scientific literature focused on use of OAT and DOACs in older persons with previous bleeding events published in the last 8 years was retrieved by the authors from the MEDLINE database using the terms "atrial fibrillation" AND "oral anticoagulant therapy", OR "new oral anticoagulants" OR "direct oral anticoagulants", AND "aged" OR "elderly" OR “older" as keywords. Reviews, recommendations and expert opinions, as well as clinical trials and large observational studies in English published until March 2019 were systematically analyzed and included according to their relevance to the objective. Additional references were obtained from the reference list of the selected full-text manuscripts.

\section{Results}

In a retrospective cohort study that enrolled $1329 \mathrm{AF}$ patients (mean age 76 years, $45 \%$ women) who developed gastrointestinal bleeding (GIB) while on anticoagulation from 2005 to 2010 , warfarin was restarted in $653(49.1 \%)$ patients [8]. Compared with patients who did not receive OAT, those who were restarted with VKAs had better cumulative survival (HR 0.67, 95\% CI: 0.56-0.81) and lower one-year cumulative incidence of thromboembolic events (HR 0.71. 95\% CI: 0.54-0.93) without a significant increase in the 90-days cumulative incidence of recurrent GIB [8]. Restarting OAT 7 to 15 days after bleeding events was associated with more favorable cumulative survival and clinical benefit including recurrent bleeding and thromboembolism [8]. A recent prospective observational cohort study on 197 consecutive patients (mean age 75 years, $60 \% \mathrm{AF}$ ) hospitalized for GIB while on OAT, investigated 90 days cumulative incidence of thromboembolic events, hospital readmissions related to GIB, and mortality in those who resumed anticoagulation and those who had anticoagulation discontinued [9]. The adjusted hazard ratios for continuing vs cessation of OAT were 0.121 (95\% CI: 0.006-0.813, p=0.03) for thromboembolism, 2.17 (95\% CI: $0.861-6.67, \mathrm{p}=0.10)$ for recurrent $\mathrm{GIB}$, and $0.632(95 \%$ CI: $0.216-1.89, \mathrm{p}=0.40$ ) for all-cause death [9]. In a retrospective Danish cohort study in the period 1996-2012 which enrolled 4602 AF patients (mean age 78 years) discharged after GIB, 27,1\% did not resume antithrombotic therapy [10]. In the whole sample, overall 2-year mortality was $39.9 \%$, major bleeding and recurrent GIB occurred in $17.7 \%$ and $12.1 \%$ of patients, respectively, whereas $12 \%$ of patients experienced a thromboembolic event. Compared with patients who did not resume OAT, all-cause mortality and thromboembolism were significantly lower either in patients who restarted OAT (HR 0.39, 95\% CI: 0.34-0.46 and HR 0.41, 95\% CI: $0.31-0.54$, respectively) and in those who restarted OAT plus antiplatelet therapy (HR 0.41, 95\% CI: 0.32-0.52 and HR 0.54, 95\% CI: $0.36-0.82$, respectively). Major bleedings, but not GIB recurrence, were significantly higher in those who restarted OAT and OAT plus antiplatelet therapy (HR 1.37, 95\% CI: 1.06-1.77, and HR 1.49, 95\% CI: 1.32-1.91, respectively) [10]. A recent retrospective analysis of medical claims data from the Truven Health Marketscan Commercial Claims and Encounters Database, from January 1, 2010, through December 31, 2014, included 1338 adults (mean age 79 years) treated with DOACs and hospitalized for GIB [11]. DOACs were restarted in 586 patients; older patients requiring blood and intensive care were less likely to restart DOAC therapy. In the whole sample resuming DOAC was not associated with 90-day thromboembolism and recurrence of GIB [11].

A recent study aimed to evaluate current clinical evidence for management of OAT after GIB, with an emphasis on whether to, when to, and how to resume an anticoagulation therapy [12]. A total of 9 studies were identified. Four retrospective cohort studies showed that resuming anticoagulation therapy was associated with significantly lower rate of thromboembolism (TE) in the general population. Meta-analyses and prospective cohort studies also supported this finding. Two retrospective cohort studies indicated an increase in GIB when anticoagulation reinitiation occurred in less than 7 days without a decrease in TE. [12]. Resuming therapy between 7 and 15 days did not demonstrate a significant increase in GIB or TE [12]. A large retrospective study showed that apixaban was associated with the significantly lowest risk of GIB compared with both rivaroxaban and dabigatran.

The Authors concluded that OAT resumption is recommended, with resumption being considered between 7 and 14 days following GIB regardless of the therapy chosen [12]. Data for warfarin management after GIB should be applied with caution to direct oral anticoagulants (DOACs) because of the quicker onset and experimental nature of reversal agents. Apixaban may be a preferred option when restarting a DOAC therapy [12]. A recent review and meta-analysis, aiming to determine the risks of recurrent GIB, thromboembolism and death in patients who resumed OAT compared to those who did not, identified 12 observational studies involving 3098 patients [13]. There was an increased risk of recurrent GIB (RR 1.91, 95\% CI 1.47-2.48, I2 =0\%, 11 studies), and a reduced risk of thromboembolism (RR $0.30,95 \%$ CI 0.13 $0.68, \mathrm{I} 2=59.8 \%, 9$ studies) and death (RR 0.51, 95\% CI 0.38-0.70, $\mathrm{I} 2=71.8 \%, 8$ studies) in patients who resumed OAC compared to those who did not. Although eleven studies were judged to be at serious risk of bias due to confounding, these results suggest that resuming OAT after OAT-related GIB appears to be associated with an increase in recurrent GIB, but a reduction in thromboembolism and death [13].

There are few observational cohort studies which investigated the clinical benefit of restarting OAT after intracranial hemorrhage (ICH). A recent review and meta-analysis concluded that reinstitution of OAT after ICH was associated with a lower risk of thromboembolic complications and a similar risk of ICH recurrence [14]. A recent study examined the timing of DOAC resumption and factors that influence decision-making in DOAC resumption in 43 patients with $\mathrm{ICH}$ who were treated with DOAC for nonvalvular atrial fibrillation before ICH onset [15]. DOAC were resumed in 19 of $39(49 \%)$ acute ICH survivors and were not resumed in 24 patients, including 4 deceased patients. The National Institutes of Health Stroke Scale score at admission tended to be higher in the no resumption group (median, 17) than in the resumption group (median, 6) $(\mathrm{p}=0.119)$. The modified Rankin Scale score was slightly poorer in the no resumption group (median, 4) than in the resumption group (median, 3$)(\mathrm{p}=0.070)$. In the resumption group, DOAC were resumed at a median of 11 days (interquartile range, 5-21 days) after ICH onset. The modified Rankin Scale score at discharge was positively correlated with the days of DOAC 
resumption $(\mathrm{R} 2=0.31, \mathrm{p}=0.013)$. The Authors concluded that early resumption of DOAC for ICH in AF patients is considered to be safe, and that the functional outcome was associated with not only resumption of DOAC but also the timing of resumption [15]. Perreault et al. investigated whether starting OAT among 683 older AF patients (mean age 83 years) after an ICH was associated with a lower risk of ischemic stroke/systemic embolism (IS/SE) and mortality but offset by an increase in major bleeding [16]. The rates (per 100 person-years) for IS/SE, death, ICH and major bleeding were $3.3,40.6,11.4$, and 2.7 for the no OAC group; and 2.6, 16.3, 5.2, and 5.2 for the OAC group, respectively. The absolute hazard ratio for IS/SE and death were 0.10 (95\% CI: 0.05 to 0.21$), 0.43$ ( $95 \%$ CI, 0.19 to 0.97 ) for recurrent ICH and 1.73 (95\% CI, 0.71 to 4.20) for major extracranial bleeding comparing OAT exposure to non-exposed. The Authors concluded that initiating OAT after ICH in older individuals with AF is associated with a reduction of IS/SE and mortality, supporting its use after ICH bleeding [16].

A recent Danish cohort study included 4541 OAT treated AF patients (mean age 81 years) experiencing traumatic injury during the period 2005-2016: $60,7 \%$ of patients resumed warfarin-based OAT and $16,7 \%$ resumed DOAC [17]. Compared with patients who did not restart OAT, resumption of warfarin or DOACs was associated with significantly lower hazards of all cause mortality (0.48, 95\% CI: $0.42-0.53$, and $0.55,95 \%$ CI: $0.47-0.66$, respectively) and ischemic stroke $(0.56,95 \% \mathrm{CI}: 0.43-0.72$, and $0.54,95 \%$ CI: $0.35-0.82$, respectively), at the cost of an increased hazard of major bleeding in those receiving VKAs $(1.30,95 \% \mathrm{CI}$ : 1.03-1.64) [17].

\section{Discussion}

The decision about whether and when to start or resume OAT following an episode of GIB must balance the risks of thromboembolism and recurrent GIB. This decision is even more complex in patients who recovered from an ICH. Most of observational studies suggest that the net clinical benefit favors resuming OAT, with a reduced risk of thromboembolism and death, despite an increase in GIB. Importantly, the clinical impact of GIB and thrombotic events are not equivalent. The case-fatality rate of OAT-related bleeding is $8-13 \%$ [18-20]. In comparison, the risk of death, institutionalization due to stroke, or disability at 3 months was $41 \%$ for all strokes in a European stroke registry [21]. Interestingly, in a retrospective cohort study in Medicare beneficiaries between January 1, 2011 and September 30, 2015 there were $1,643,123$ patients with 1,713,183 new episodes of oral anticoagulant treatment (mean age 76.4 years) [22]. Among patients initiating OAT, incidence of hospitalization for upper GIB was highest in patients prescribed rivaroxaban and the lowest in patients prescribed apixaban. For each anticoagulant the incidence of hospitalization for upper GIB was lower among patients who were receiving proton pump inhibitor co-therapy [22].

However, most of the evidence supporting the initiation or reinitiation of OAT after a major bleeding comes from observational cohort studies which may be heavily flawed by several limitations. These observational studies are highly heterogeneous in the pooled estimates for thromboembolism and mortality. More important, all these studies shared a high potential risk of bias due to baseline confounding. Possible confounders included age, indication for OAT, source of bleeding, risk of thrombosis, risk of recurrent bleeding, and comorbidities. In the absence of randomization, differences in baseline characteristics which are prognostic for outcomes may have influenced the decision whether or not resuming OAT. Therefore, the mortality benefit observed in most of these studies might be at least in part accounted for by a higher prescription of OAT to patients with better general health status. Moreover, in all of these studies involving older AF patients there is no mention of some comprehensive geriatric assessment, even if there is strong evidence that geriatric syndromes may heavily affect either OAT use and survival in these patients [23-27]. Therefore, in the absence of high-quality data regarding the optimal timing of resumption and type of OAT in this setting, decision-making should be individualized with discussion about the risks and benefits and incorporating patient values and preferences.

The European Society of Cardiology Working Group on Thrombosis has recently released an expert consensus focused on the management of antithrombotic therapy in AF patients after a bleeding event [28]. Patients with AF were categorized according to the estimated thrombotic risk as very high (including those with $\mathrm{CHA}_{2} \mathrm{DS}_{2}$-VASC score $\geq 6$, or mechanical mitral valves or cardiac assist devices), high (including those with $\mathrm{CHA}_{2} \mathrm{DS}_{2}$-VASC score $=4-5$ or a mechanical aortic bileaflet valve) and moderate $\left(\mathrm{CHA}_{2} \mathrm{DS}_{2}\right.$-VASC score $\left.=2-3\right)$ [28]. Similarly, an expert consensus panel of the American College of Cardiology identified high thrombotic risk in AF patients as the presence of at least one of $\mathrm{CHA}_{2} \mathrm{DS}_{2}$-VASC score $\geq 6$, stroke/Transient Ischemic Attack within 3 months, stroke risk $\geq 10 \%$ year, rheumatic valve disease or mitral stenosis [29]. The European expert panel also provided a consensus definition of recurrent bleeding risk categories, including a very high-risk group (ICH where no treatment is possible or effective, and life-threatening extracranial bleeding where the source of bleeding is either not identified or identified but not treated effectively), and a high risk group (major extracranial bleeding where the source is identified but not treated effectively and definitively) [28]. Clinical decisions are extremely complex in patients fulfilling either very high- or high-risk features for thrombotic and bleeding events. On this background, flow-charts for (re)-initiation of OAT after GIB and ICH were provided [28,29], including left atrial appendage occlusion for patients deemed at high or very high thrombotic and bleeding risks. In this context, we believe that older age per se should not be considered the best factor to be considered among variables for the net assessment in favour of withholding OAT in elderly people: rather, a careful geriatric assessment may provide physicians a more reliable evaluation of the net clinical benefit of OAT in these elderly patients [30-32].

In conclusion, the decision about whether or not to start or reinitiate OAT in older AF patients with a previous bleeding should be individualized and based on a careful evaluation of thrombotic and bleeding risks, with discussion about the risks and benefits and incorporating patient values and preferences. In this setting, a thorough comprehensive geriatric assessment of general health status and estimated survival may assist physicians in the decisionmaking for older complex patients.

\section{References}

1. Ruff CT, Giugliano RP, Braunwald E, et al. Comparison of the efficacy and safety of new oral anticoagulants with warfarin in patients with atrial fibrillation: a meta-analysis of randomized trials. Lancet 2014;383:955-62.

2. Held C, Hylek EM, Alexander JH, et al. Clinical outcomes and management associated with major bleeding in patients with atrial fibrillation treated with apixaban or warfarin: insights 
from the ARISTOTLE trial. Eur Heart J 2015;36:1264-72.

3. Andreotti F, Rocca B, Husted S, et al. Antithrombotic therapy in the elderly: expert position paper of the European Society of Cardiology Working Group on Thrombosis. Eur Heart J 2015;36:3238-49.

4. Vanessa S, Andrew K, Katrina p, et al. Annual risk of major bleeding among persons without cardiovascular disease not receiving antiplatelet therapy. JAMA 2018;319:2507-20.

5. Kooistra HA, CalfAH, Piersma-Wichers M, et al. Risk of bleeding and thrombosis in patients 70 years or older using Vitamin K antagonists. JAMA Intern Med 2016;176:1176-83.

6. Poli D, Antonucci E, Testa S, et al. Bleeding risk in very old patients on vitamin $\mathrm{K}$ antagonist treatment. Results of a prospective collaborative study on elderly patients followed by Italian centres for anticoagulation. Circulation 2011;124:824-9.

7. Lip GY, Lane DA. Assessing bleeding risk in atrial fibrillation with the HAS-BLED and ORBIT scores: clinical application requires focus on the reversible bleeding risk factors. Eur Heart J 2015;36:3265-7.

8. Wagas Q, Chetan M, Iani P, et al. Restarting anticoagulation and outcomes after major gastrointestinal bleeding in atrial fibrillation. Am J Cardiol 2014;113:662-8.

9. Sengupta N, Feuerstein JD, Patwardhan VR, et al. The risks of thromboembolism vs. recurrent gastrointestinal bleeding after interruption of systemic anticoagulation in hospitalized inpatients with gastrointestinal bleeding: a prospective study. Am. J. Gastroenterol 2015;110:328-35.

10. Staerk L, Lip GYH, Olesen JB, et al. Stroke and recurrent haemorrhage associated with antithrombotic treatment after gastrointestinal bleeding in patients with atrial fibrillation: nationwide cohort study. BMJ 2015 351:5876.

11. Sengupta N, Marshall L.A, Ham S, et al. Rebleeding vs thromboembolism after hospitalization for gastrointestinal bleeding in patients on direct oral anticoagulants. Clin Gastroenterol Hepatol 2018;16:1893-900.

12. Kido K, Scalese MJ. Management of oral anticoagulation therapy after gastrointestinal bleeding: whether to, when to, and how to restart an anticoagulation therapy. Ann Pharmacother 2017;51:1000-9.

13. Little D, Chai-Adisaksopha C, Hillis C, et al. Resumption of anticoagulant therapy after anticoagulant-related gastrointestinal bleeding: A systematic review and meta-analysis. Thromb Res 2019; 175:102-9.

14. Murthy SB, Gupta A, Merkler AE, et al. Restarting anticoagulant therapy after intracranial hemorrhage: a systematic review and meta-analysis. Stroke 2017;48:1594-600.

15. Kato Y, Hayashi T, Suzuki K, et al. Resumption of direct oral anticoagulants in patients with acute spontaneous intracerebral hemorrhage. J Stroke Cerebrovasc Dis 2019;28:104292.

16. Perreault S, Coté R, White-Guay B, et al. Anticoagulants in older patients with nonvalvular atrial fibrillation after intracranial hemorrhage. J Stroke 2019;21:195-206.

17. Staerk L, Fosbol EL, Lamberts M, et al. Resumption of oral anticoagulation following traumatic injury and risk of stroke and bleeding in patients with atrial fibrillation: a nationwide cohort study. Eur Heart J 2018;39:1698-705.

18. Linkins LA, Choi PT, Douketis JD. Clinical impact of bleeding in patients taking oral anticoagulant therapy for venous thromboembolism: a meta analysis. Ann Intern Med 2003; 139:893-900.

19. Carrier M, Le Gal G, Wells PS, et al. Systematic review: casefatality rates of recurrent venous thromboembolism and major bleeding events among patients treated for venous thromboembolism. Ann Intern Med 2010;152:578-9.

20. Douketis JD, Arneklev K, Goldhaber SZ, et al. Comparison of bleeding in patients with nonvalvular atrial fibrillation treated with ximelagartran or warfarin: assessment of incidence, casefatality rate, time course and sites of bleeding and risk factors for bleeding. Arch Intern Med 2006;166:853-9

21. Heuschmann PU, Wiedmann S, Wellwood I, et al. Three-month stroke outcome: the european registers of strokes (EROS) investigators. Neurology 2011;76:159-65.

22. Ray WA, Chung CP, Murray KT, et al. Association of oral anticoagulants and proton pump inhibitor cotherapy with hospitalization for upper gastrointestinal tract bleeding. JAMA 2018;320:2221-30.

23. Savarese G, Sartipy U, Friberg L, et al. Reasons for and consequences of oral anticoagulant underuse in atrial fibrillation with heart failure. Heart 2018;104:1093-100.

24. Bo M, Sciarrillo I, Maggiani G, et al. Health status, geriatric syndromes and prescription of oral anticoagulant therapy in elderly medical inpatients with atrial fibrillation. Geriatr Gerontol Int 2017; 17:416-23.

25. Mazzone A, Bo M, Lucenti A, et al. The role of comprehensive geriatric assessment and functional status in evaluating the patterns of antithrombotic use among older people with atrial fibrillation. Arch Gerontol Geriatr 2016;65:248-54.

26. Graham DJ, Baro E, Zhang R, et al. Comparative stroke, bleeding, and mortality risks in older medicare patients treated with oral anticoagulants for nonvalvular atrial fibrillation. Am J Med 2019;132:596-604.

27. Sinnaeve PR, Brueckmann M, Clemens A, et al. Stroke prevention in elderly patients with atrial fibrillation: challenges for anticoagulation. J Intern Med 2012;271:15-24.

28. Halvorsen S, Storey RF, Rocca B, et al. Management of antithrombotic therapy after bleeding in patients with coronary artery disease and/or atrial fibrillation: expert consensus paper of the European society of cardiology working group on thrombosis. Eur Heart J 2017;38:1455-62.

29. Steffel J, Verhamme P, Potpara TS, et al. The 2018 European Heart rhythm Association practical guide on the use of nonvitamin $\mathrm{K}$ antagonist oral anticoagulants in patients with atrial fibrillation. Eur Heart J 2018;39:1330-93.

30. Nishimura RA, Otto CM, Bonow RO, et al. 2017 AHA/Acc Focused Update of the 2014 AHA/ACC. Guideline for the management of patient with valvular heart disease. A report of the American College of Cardiology/American Heart Association task force on clinical practice guidelines. Circulation 2017;135:e1159-95.

31. Baldasseroni S, Bo M, Brambati T, et al. [How much frailty is important in cardiology?].[Article in Italian]. G Ital Cardiol (Rome) 2019;20:210-22.

32. Bo M, Marchionni N. Practical use of direct oral anticoagulants in the older persons with atrial fibrillation. Eur J Intern Med 2019;71:32-8. 